\section{Quetiapine-Induced Hypersalivation}

Sir: Quetiapine fumarate is a dibenzothiazepinederivative antipsychotic with combined antagonism at dopamine $\left(\mathrm{D}_{1}, \mathrm{D}_{2}\right)$ and serotonin type 1 and type $2\left(5-\mathrm{HT}_{1 \mathrm{~A}}\right.$, $\left.5-\mathrm{HT}_{2 \mathrm{~A}}, 5-\mathrm{HT}_{2 \mathrm{C}}\right)$ receptors. Additionally, quetiapine antagonizes $\alpha_{1}$ - and $\alpha_{2}$-adrenergic and histamine $\mathrm{H}_{1}$ receptors. Protean binding at the above receptors explains the common side effects, which include hypersomnolence, hypotension, headache, weight gain, and gastrointestinal disturbance. However, to our knowledge, there have been no case reports of quetiapine-induced hypersalivation. We report the case of a patient with schizoaffective disorder taking low-dose quetiapine who developed hypersalivation, which remitted after discontinuation of the drug. We suggest that quetiapine antagonism at the $\alpha_{2}$ receptor contributes to the phenomenon of hypersalivation.

Case report. Ms. A, a 36-year-old woman of Korean descent with a 16-year history of schizoaffective disorder, was admitted to the adult psychiatric medical care unit with a relapse of mania with psychosis. The patient's symptoms included elated mood, grandiose delusions of being a Power Ranger, disorganized behavior, and decreased need for sleep. The patient had been previously stabilized on clozapine with the only noted side effect being increased sedation. During this hospitalization, the patient refused administration of clozapine and instead was started on risperidone. Within a few days of commencement, the patient developed extrapyramidal symptoms with rigidity, dystonia, and slurring of speech, which prompted discontinuation of the risperidone. After a drug-free period of 3 days, the patient was started on quetiapine $25 \mathrm{mg}$ b.i.d., and within a day, she developed increased salivation. When the dosage was increased to $50 \mathrm{mg}$ b.i.d., the salivation worsened; the patient was drooling most of the day, and all of her clothing was wet. Benztropine (1 mg p.o. b.i.d.) and atropine eye drops $(0.1 \%)$ sublingually did not reduce the hypersalivation. The quetiapine was stopped, and 1 day later, the hypersalivation resolved.

Based on the temporal relationship between the administration and discontinuation of quetiapine and the onset and cessation of the patient's hypersalivation, it is possible that the hypersalivation was induced by the quetiapine. Hypersalivation is a common and distressing side effect seen with many psychotropic medications. However, no previous reports have associated increased salivation with the use of quetiapine. We attempt to explain the mechanism behind this event using the drug's known receptor affinities and the resultant binding effects observed in a closely related antipsychotic agent.

Among the antipsychotics, clozapine is well recognized for inducing increased, voluminous salivation early in the course of treatment in up to $54 \%$ of patients. ${ }^{1}$ Clozapine is structurally similar to quetiapine and possesses a comparably broad receptor profile, which includes antagonistic activity at dopamine $\left(\mathrm{D}_{1}, \mathrm{D}_{2}, \mathrm{D}_{3}, \mathrm{D}_{4}\right)$ receptors, adrenergic blockade activity at the $\alpha_{1}$ and $\alpha_{2}$ receptors, and antagonism at serotonin 5- $\mathrm{HT}_{2}$ receptors and histamine $\mathrm{H}_{1}$ receptors. However, unlike quetiapine, clozapine possesses both agonistic and antagonistic properties at muscarinic receptors. Specifically, clozapine has antagonist activity at $\mathrm{M}_{1}, \mathrm{M}_{2}, \mathrm{M}_{3}$, and $\mathrm{M}_{5}$ receptors, but exhibits agonist effects on the $\mathrm{M}_{4}$ receptor. One proposed mechanism for clozapine-induced hypersalivation focuses on its antagonism of $\alpha_{2}$-adrenergic receptors. ${ }^{2}$ Through blockade of sympathetic stimulation, parasympathetic stimulation is left unopposed to cause high salivary flow rates. Direct parasympathetic stimulation through its agonist effects at the $\mathbf{M}_{4}$ receptor has also been suggested to explain clozapine-induced hypersalivation. ${ }^{3}$

Quetiapine and clozapine both share blocking affinity at $\alpha_{2}$-adrenergic receptors, whereas only clozapine has agonist properties at the $\mathrm{M}_{4}$ receptor. We thus propose that $\alpha_{2}$-adrenergic blockade is the mechanism for quetiapineinduced hypersalivation. Further study is needed to draw definite conclusions regarding this proposed relationship.

The authors report no financial affiliation or other relationships relevant to the subject of this letter.

\section{REFERENCES}

1. Davydov L, Botts SR. Clozapine-induced hypersalivation. Ann Pharmacother 2000;34:662-665

2. Berlan M, Montastruc JL, Lafontan M. Pharmacological prospects for alpha 2-adrenoceptor antagonist therapy. Trends Pharmacol Sci 1992;13:277-282

3. Zorn SH, Jones SB, Ward KM, et al. Clozapine is a potent and selective muscarinic M4 receptor agonist. Eur J Pharmacol 1994;269:R1-R2

Shannon Allen, B.S. Zachary Hoffer, Ph.D. Maju Mathews, M.D., M.R.C.Psych. Drexel University College of Medicine Philadelphia, Pennsylvania

\section{Sleep-Related Hypomanic Symptoms as a Predictor of Bipolar Spectrum Disorders}

Sir: Deciding whether to prescribe antidepressants for the depressed patients with bipolar spectrum disorders remains a challenge for clinicians. Two most-feared potential consequences of using antidepressants in patients with bipolar spectrum disorders are triggering a manic switch and/or generating a rapid cycling course of the disease. ${ }^{1}$ Experts have long tried to recognize the depressed patients who are at a greater risk for switching to mania; thus, several symptoms have been suggested as "soft signs" of bipolarity, including hypersomnia, psychomotor retardation, young age at onset of depression, family history of bipolar disorder, drug-induced hypomania, seasonality of the episodes, and diurnal variation of the symptoms. ${ }^{2}$ However, the list is not yet complete, and many cases still cannot be correctly predicted. As a result, clinical findings that could be used as new predictors of bipolar spectrum disorders could be helpful for future interventions.

Case report. Ms. A was a 27-year-old married woman who experienced an aggravation of depressive and anxiety symptoms for 6 months beginning in August 2005. Symptoms included depressed mood, loss of interest, repeated crying spells, loss of concentration, insomnia, decreased appetite, irritability, worry, and preoccupation with death. 
Past history revealed no significant finding other than mild depressive and anxiety symptoms in the previous 4 years and no episodes of mania or hypomania.

She had no suggestive symptoms for a bipolar spectrum disorder except for very brief periods (about 30 seconds) of euphoria and inflated self-esteem, which occurred just before falling asleep, in a semiconscious state between wakefulness and sleep. During these periods, she experienced an exuberant sense of power, and she believed that she was able to "move mountains." Family history for bipolar disorder was negative.

The patient met the criteria for major depressive disorder based on DSM-IV-TR and received $5 \mathrm{mg} /$ day of citalopram (February 2006), and after 5 days, the dose was increased to 10 $\mathrm{mg} /$ day, in addition to $1.25 \mathrm{mg}$ /day of alprazolam, in 3 divided doses. One week later, the patient showed significant improvement. In addition, alprazolam had reduced the sleep latency, and the patient did not experience or remember any sleep-related hypomanic symptoms. Instead, she reported having distinct periods of euphoria and talkativeness 15 to 20 minutes after taking each dose of alprazolam. These periods would happen after all doses of alprazolam and would completely resolve after about 10 minutes, and the patient would return to her depressed baseline. The patient did not experience any other side effects other than a mild sedation during the day. The alprazolam dose was reduced to $0.75 \mathrm{mg} / \mathrm{day}$ to decrease sedation and then tapered to complete cessation in the next 3 weeks, and citalopram was continued. Sleep-related hypomanic symptoms did not recur after reducing or discontinuing alprazolam. The patient was followed for 6 more months and remained euthymic (August 2006).

Recent studies suggest that the lifetime prevalence of bipolar spectrum disorders in the general population is more than $6 \%,{ }^{3}$ that is, much higher than what was previously speculated. ${ }^{2}$ It is also reported that about $12.5 \%$ of the patients initially diagnosed as having depression will be reclassified as having a bipolar spectrum disorder in their course of illness. ${ }^{4}$

This patient reported experiencing distinct brief periods of euphoria and inflated self-esteem before sleep onset, which could potentially be related to bipolar spectrum disorders. Even though we did not have enough evidence to accept it as a new soft sign of bipolar spectrum disorders before starting the medications, transient hypomanic symptoms induced by taking alprazolam suggested that "sleep-related periods of euphoria" could be assumed as one of the predictors of bipolar spectrum disorders.

We are aware that had the patient been followed without decreasing the dose of alprazolam, she might not have developed a bipolar disorder. Obviously, this could not be performed because of ethical considerations. Future studies could tell more about the diagnostic value and effect of each of the soft signs on the long-term course of the disease.

The authors report no financial or other affiliation relevant to the subject of this letter.

\section{REFERENCES}

1. Gijsman HJ, Geddes JR, Rendell JM, et al. Antidepressants for bipolar depression: a systematic review of randomized, controlled trials. Am J Psychiatry 2004;161:1537-1547

2. Katzow JJ, Hsu DJ, Ghaemi SN. The bipolar spectrum: a clinical perspective. Bipolar Disord 2003;5:436-442

3. Judd L, Akiskal H. The prevalence and disability of bipolar spectrum disorders in the US population: re-analysis of the database taking into account the subthreshold cases. J Affect Disord 2003;73:123-131
4. Akiskal HS, Maser JD, Zeller PJ, et al. Switching from 'unipolar' to bipolar II: an 11-year prospective study of clinical and temperamental predictors in 559 patients. Arch Gen Psychiatry 1995;52:114-123

Seyed Vahid Shariat, M.D. Amir Shabani, M.D. Tehran Psychiatric Institute Mental Health Research Center Iran University of Medical Sciences

Tehran, Iran

\section{A Case of Obsessive-Compulsive Disorder Responding to Duloxetine}

Sir: Despite extensive clinical use of serotonin reuptake inhibitors, clomipramine, and venlafaxine ${ }^{1}$ in the treatment of obsessive-compulsive disorder (OCD), to our knowledge there have been no reports on the use of duloxetine to treat patients with this disorder. We report the successful use of duloxetine to treat a patient with OCD.

Case report. Mr. A, a 38-year-old man, had an 8-year history of OCD (DSM-IV criteria), which began abruptly several months before his wedding and was characterized by senseless and recurrent thoughts that caused marked distress, such as, "Why do doors open and close?" and "Why does water fall out when a bucket turns upside down?" He felt compelled to repeatedly hit a door with his hands to relieve his distress; relief was only transient despite hitting the door all day long. The symptoms led to impaired work and social functioning. Treatment was begun in 1998 with clomipramine, $75 \mathrm{mg} /$ day, and clonazepam, $3 \mathrm{mg} /$ day, in addition to supportive psychotherapy. After 1 month of treatment, Mr. A had experienced significant symptomatic relief, though he had also developed loss of libido, retarded ejaculation, and anorgasmia. Clomipramine and clonazepam were discontinued, and he began treatment with paroxetine $(60 \mathrm{mg} / \mathrm{day})$, which he did not tolerate due to severe headaches, retarded ejaculation, and anorgasmia. Mirtazapine, $30 \mathrm{mg} /$ day, was started to alleviate his sexual complaints.

For the next 4 years, his mild obsessional thoughts did not interfere with his daily activities. Without warning, Mr. A experienced the onset of sexual obsessions in 2003 that were extremely distressing and offended his moral sense. They were characterized by a recurrent impulse to stare at attractive women. This impulse was associated with an urgent need to change his facial expression (involving smiling, grimaces, or other facial contortions), which led to considerable anxiety. These symptoms led to social withdrawal and affected his normal routines. His antidepressant treatment was switched from mirtazapine to escitalopram ( $20 \mathrm{mg} /$ day $)$. He experienced mild improvement, which allowed him to return to his routine activities.

During the next 12 months, he was again bothered with persistence and worsening of his symptoms, experiencing 20 to 30 "crises" a day. With his consent, escitalopram treatment was discontinued and treatment with duloxetine $(60 \mathrm{mg} /$ day $)$ was initiated. After 4 weeks and no symptom improvement, the dose was boosted to $120 \mathrm{mg}$ /day. One month later, Mr. A had achieved remarkable symptom improvement, reporting no subsequent obsessional "crises." During a 1-year follow-up with duloxetine maintenance, he continued to do well. According to Mr. A, this is the longest period he had ever had free of intrusive obsessional thoughts. No side effects were reported. 
Reports have implicated noradrenergic as well as the serotonergic neurotransmitter systems in the genesis of OCD. ${ }^{2}$ For that reason, duloxetine, with its balanced serotoninnorepinephrine reuptake inhibition properties, may be worthwhile to study in the treatment of OCD.

Dr. Blay has been a speaker for Boehringer Ingelheim and Eli Lilly. Dr. Black has been a consultant to and has been on the speakers or advisory boards for Shire, Pfizer, and Forest; has received grant/research support from Shire and Forest; and has received honoraria from Shire and Pfizer.

\section{ReferenCes}

1. Denys D, van der Wee N, van Megen HJ, et al. A double blind comparison of venlafaxine and paroxetine in obsessive-compulsive disorder. J Clin Psychopharmacol 2003;23:568-575

2. Oades RD, Ropcke B, Egger C. Monoamine activity reflected in urine of young patients with obsessive compulsive disorder, psychosis with and without reality distortion and healthy subjects: an explorative analysis. J Neural Transm Gen Sect 1994;96:143-159

Sergio Luís Blay, Ph.D. Department of Psychiatry São Paulo School of Medicine Federal University of São Paulo UNIFESP São Paulo, Brazil

Donald W. Black, Ph.D.

Roy J. and Lucille A. Carver College of Medicine University of Iowa Iowa City, Iowa

\section{Absence of Cardiac Side Effects During Treatment of Schizophrenia With Risperidone in a Patient With Wolff-Parkinson-White Syndrome}

Sir: Excessive prolongation of the QT interval can predispose to a peculiar ventricular arrhythmia called torsade de pointes, or sudden cardiac death. QT prolongation has been found to occur with some older conventional and newer atypical antipsychotics. ${ }^{1}$ Nevertheless, the recent literature suggests that atypical antipsychotics are not associated with torsade de pointes except in the case of a concomitant risk factor (e.g., polypharmacy, overdose, restraint, substance misuse, electrolyte imbalance). ${ }^{1,2}$

The risk for QT prolongation does not seem to be the same for all atypical antipsychotics. Olanzapine seems to present a quasi-inexistent risk $^{1}$; risperidone, quetiapine, aripiprazole, and clozapine present weak to moderate risks ${ }^{2}$; and ziprasidone presents higher risks. ${ }^{1}$ Sertindole has been associated with QT prolongation leading to fatal cardiac arrhythmias.

Other cardiovascular risks associated with antipsychotic use include cardiac factors such as long QT syndromes, ischemic heart disease, myocarditis, and sinus bradycardia. ${ }^{3}$ A case report of olanzapine-induced corrected QT (QTc) prolongation in a patient with Wolff-Parkinson-White (WPW) syndrome has recently been published. ${ }^{4}$

We report the clinical case of a 35-year-old man with DSM-IV schizophrenia and WPW syndrome who received treatment with risperidone.

Case report. In May 2004, Mr. A was hospitalized due to the exacerbation of paranoid delusions and auditory hallucinations. Because of compliance issues, we decided to administer long- acting injectable risperidone, $50 \mathrm{mg}$, allied with an oral treatment of risperidone, $2 \mathrm{mg} / \mathrm{day}$, during the first month. Concomitant treatment consisted of trazodone, $100 \mathrm{mg} / \mathrm{day}$, and lorazepam, $15 \mathrm{mg} /$ day. Before the start of treatment, the QTc interval was $420 \mathrm{~ms}$. One week later, it was at $452 \mathrm{~ms}$ and 2 weeks later, $469 \mathrm{~ms}$. A second injection of risperidone, $50 \mathrm{mg}$, was then administered. Subsequently, the patient refused further injections. Oral treatment with risperidone, $6 \mathrm{mg} / \mathrm{day}$, was then administered. One month later, his psychotic symptoms improved, and his QTc interval was $472 \mathrm{~ms}$.

Life-threatening ventricular arrhythmias in WPW syndrome are related to a rapid anterograde conduction throughout the bypass tract and are not related to torsade de pointes. Mr. A exhibited paroxysmal palpitations, and the baseline electrocardiogram showed intermittent pre-excitation from a left lateral accessory bypass tract. Pre-excitation regressed during sinus tachycardia with an enhanced conduction in the normal conduction system, suggesting a long refractory period within the bypass tract and a low risk for life-threatening ventricular arrhythmias.

In conclusion, WPW syndrome per se seems not to be a contraindication for atypical antipsychotics, and standard safety measures such as the regular measurement of the QTc interval and the avoidance of concomitant QT-prolonging drugs should be sufficient precautions when treating WPW patients with atypical antipsychotics.

The authors report no financial or other relationship relevant to the subject of this letter.

\section{REFERENCES}

1. Glassman AH. Schizophrenia, antipsychotic drugs, and cardiovascular disease. J Clin Psychiatry 2005;66(suppl 6):5-10

2. Vieweg WVR. New generation antipsychotic drugs and QTc interval prolongation. Prim Care Companion J Clin Psychiatry 2003;5: 205-215

3. Gury C, Canceil O, Iaria P. Antipsychotiques et sécurité cardiovasculaire: données actuelles sur les allongements de l'intervalle QT et le risque d'arythmies ventriculaires. Encéphale 2000;26:62-72

4. Su KP, Chuang CL, Chen KP, et al. Olanzapine-induced QTc prolongation in a patient with Wolff-Parkinson-White syndrome. Schizophr Res 2004;66:191-192

Fabrice Bon, M.D.

Eric Louis Constant, M.D., Ph.D.

Department of Psychiatry, Saint-Luc University Clinics

Peter Goethals, M.D.

Department of Cardiology, Saint-Jean Clinic

Daniel Neu, M.D.

Department of Psychiatry, Erasme University Hospital Brussels, Belgium

\section{A Case of Late-Onset Pedophilia and Response to Sertraline}

Sir: Pedophilia is a psychiatric disorder in a person at least 16 year old that is characterized by sexually arousing fantasies, sexual urges, or behaviors involving sexual activity with a prepubescent child (aged 13 years or younger). ${ }^{1}$ These fantasies are acted out and cause marked distress or interpersonal difficulties. Pedophilia is a common and often overlooked syndrome that 
risks the child victim's well-being and further psychosocial development and adaptive functioning. While a majority of cases of pedophilia begin among adolescents, ${ }^{2}$ in the elderly it is relatively rare and confined to a few case reports. ${ }^{3-5}$

In the present report, we describe a patient with emergence of pedophilia in late life, the assessment, and response to treatment with sertraline.

Case report. Mr. A, a 70-year-old man, was hospitalized in 2005 with a history of engaging in sexual behavior with female children 6 to 7 years old. The history was obtained from Mr. A's adult son, as the patient initially denied any problem. During the last 13 years, Mr. A had been giving young girls money, later taking them to remote places and undressing them and fondling their genitals. On 1 or 2 occasions, he had apparently attempted intercourse (based on reports from the victims' families of vaginal bleeding). No legal complaint had been filed by the victims' families. However, Mr. A's family was concerned that he may molest these children and get arrested and thus locked him in the house often. He is married and heterosexually oriented and was living with his wife and children. The patient reported no recent change in the sexual relationship with the wife, which was corroborated by her. Mr. A's medical history was notable for a cataract surgery in the left eye 3 months before admission. He had no history of psychiatric or developmental problems and had exhibited no prior deviant sexual behavior. There was no history suggestive of cerebrovascular disease or dementia.

On examination, Mr. A was cooperative and had good eyeto-eye contact. His thought processes were logical and clear. He denied any hallucinations or delusions and appeared to be euthymic. He was alert and attentive and scored 29 of 30 on the modified Mini-Mental State Examination, ${ }^{6}$ missing an item in attention and concentration. Results of repetition, naming, memory, and constructional tasks were within normal limits. His ability to perform simple constructions was also within normal limits, and he had no difficulty with an alternating motor patterns test. Mr. A's neuropsychological assessment showed deficits in focused attention, design fluency, and visual working memory. His abstract ability was within normal limits. Physical examination, including detailed neurologic examination, found no abnormalities. Results of laboratory tests, including liver, thyroid, and renal function; hemogram; electrocardiogram; serum Veneral Disease Research Laboratory test; ELISA for human immunodeficiency virus; and serum testosterone levels (measured by chemiluminescent immunoassay), were all in the normal range. Structural magnetic resonance imaging of the brain showed findings within normal limits.

After a couple of weeks of inpatient stay, Mr. A corroborated his sexual attraction to young girls and recognized that this was a potential problem for him in the village. He fulfilled DSMIV-TR criteria for pedophilia. ${ }^{1}$ He was started on sertraline, 50 $\mathrm{mg} /$ day, which was increased to $100 \mathrm{mg}$ /day after 3 weeks. He also became engaged in individual counseling, which initially involved establishing a therapeutic alliance and gaining the patient's confidence. With this regimen and increased supervision, Mr. A had a significant behavioral improvement with no reports of similar incidents during the next 1 year of follow-up and reported a decrease in desire on a visual analogue scale.

The index patient presented with late-onset sexual activity with prepubescent girls in the absence of schizophrenic, affective, obsessive, or dementia symptoms. In contrast to the typically reported cases of pedophilia, the index patient had an unusual presentation with emergence of pedophilia in late life.
The etiology of pedophilia is unknown. Paraphiliac behavior has been reported secondary to temporal lobe epilepsy, postencephalitic neuropsychiatric syndromes, septal lesions, frontal lobe lesions, bilateral temporal lobe lesions, multiple sclerosis, and tumors in various sites. ${ }^{7}$ Recent reports have noted the concurrence of emergence of pedophilia in late life and brain disease. One report described 2 patients with pedophilia who had temporal lobe hypometabolism as revealed by positron emission tomography; 1 patient had frontotemporal dementia and the other had bilateral hippocampal sclerosis. ${ }^{4}$ No evidence of structural brain lesion, however, was evident in our patient.

Management of pedophilia at any stage poses a serious clinical challenge. Antiandrogens and hormonal agents such as estrogen, medroxyprogesterone acetate, cyproterone acetate, and gonadotropin analogues have been used; however, the effectiveness of treatment was reduced because of the various adverse reactions, ${ }^{7}$ and only a minority of subjects are likely to take an antiandrogen on their own. Several recent reports suggest successful treatment of paraphilias with selective serotonin reuptake inhibitors, e.g., sertraline, fluoxetine, and fluvoxamine. ${ }^{8-10}$ However, the efficacy of these drugs in the treatment of pedophilia emerging in late life is not known. The case reported here suggests the efficacy of sertraline in pedophilia. In the elderly, it is critical to evaluate for underlying structural and functional brain abnormalities.

The authors report no financial or other relationship relevant to the subject of this letter.

\section{REFERENCES}

1. American Psychiatric Association. Diagnostic and Statistical Manual of Mental Disorders, Fourth Edition, Text Revision. Washington, DC: American Psychiatric Association; 2000

2. McConaghy N. Paedophilia: a review of the evidence. Aust N Z J Psychiatry 1998;32:252-265

3. Kurland ML. Pedophilia erotica. J Nerv Ment Dis 1960;131:394-403

4. Mendez MF, Chow T, Ringman J, et al. Pedophilia and temporal lobe disturbances. J Neuropsych Clin Neurosci 2000;12:71-76

5. Burns JM, Swerdlow RH. Right orbitofrontal tumor with pedophilia symptom and constructional apraxia sign. Arch Neurol 2003;60: 437-440

6. Folstein MF, Folstein SE, McHugh PR. "Mini-mental state": a practical method for grading the cognitive state of patients for the clinician. J Psychiatr Res 1975;12:189-198

7. Bradford JM. The neurobiology, neuropharmacology, and pharmacological treatment of the paraphilias and compulsive sexual behavior. Can J Psychiatry 2001;46:26-34

8. Greenberg DM, Bradford JM, Curry S, et al. A comparison of treatment of paraphilias with three serotonin reuptake inhibitors: a retrospective study. Bull Am Acad Psychiatry Law 1996;24:525-532

9. Kafka MP. Sertraline pharmacotherapy for paraphilias and paraphilia-related disorders: an open trial. Ann Clin Psychiatry 1994;6:189-195

10. Stein DJ, Hollander E, Anthony DT, et al. Serotonergic medications for sexual obsessions, sexual addictions, and paraphilias. J Clin Psychiatry 1992;53:267-271

Naren Prahlada Rao, M.B.B.S. National Institute of Mental Health and Neurosciences Bangalore, India

Prabhat K. Chand, M.D., D.N.B. Department of Psychiatry Kasturba Medical College Manipal, India

Pratima Murthy, D.P.M., M.D. National Institute of Mental Health and Neurosciences Bangalore, India 


\section{Risperidone-Induced Neuroleptic Malignant Syndrome in Neurodegenerative Disease: A Case Report}

Sir: Neuroleptic malignant syndrome (NMS) is uncommon with the use of atypical antipsychotics. ${ }^{1}$ This report describes the case of a 34-year-old male with suspected neurodegenerative disease who developed this complication with the use of risperidone. He was successfully treated with dantrolene and bromocriptine and was discharged in stable condition after 7 days.

Case report. Mr. A is a 34-year-old white man known to have mild mental retardation (IQ estimate, 68). He was born through a first-degree consanguineous relationship. Prior to the illness reported here, he lived by himself and was able to perform activities of daily living without assistance or any medications. Eleven months ago, he started to develop slowing of movements. He was thoroughly investigated and diagnosed at that time with acute parkinsonism and was started on treatment with carbidopa/levodopa and benztropine. Mr. A continued to show progressive cognitive deterioration and incontinence of bowel and bladder over the next 8 months and was moved to a nursing home 3 months ago. In addition, he began to have symptoms suggestive of major depression during the past 6 months and was started on paroxetine. Following improvement in his affective symptoms, paroxetine was gradually tapered and discontinued after 5 months. However, he began to develop psychotic features and was started on risperidone at a dose of $4 \mathrm{mg}$ daily 3 weeks prior to his admission to our hospital. Within 48 hours of starting risperidone, he developed generalized muscle rigidity. However, risperidone was continued over the next 3 weeks until he developed hyperpyrexia and was admitted to a nearby hospital. He was treated there with antibiotics for 2 days, with a presumptive diagnosis of sepsis. Blood cultures, cerebrospinal fluid examination, and computed tomographic (CT) scan of the head showed no abnormal findings. Subsequently, he was transferred to our hospital, as he showed no improvement.

Upon admission to our hospital, Mr. A was hyperthermic $\left(104.4^{\circ} \mathrm{F}\right.$ ), hypertensive (blood pressure, $138 / 79 \mathrm{~mm} \mathrm{Hg}$ ), tachycardic (heart rate, 146 beats/min), tachypneic (respiratory rate, 28 breaths/min), and unresponsive. He had a startled facial expression and was drooling. Mr. A maintained a decorticate posture and had lead-pipe rigidity in all limbs. Eye movements were conjugate, and pupils were equal and reactive to light. Deep tendon reflexes were brisk and symmetric. Primitive reflexes and jaw jerk were also present. Toes were up-going bilaterally. The remainder of his physical examination was unremarkable.

Blood tests showed leukocytosis, but electrolytes were within the normal range. He was given a presumptive diagnosis of NMS in accordance with DSM-IV criteria. Risperidone and antibiotics were discontinued, and prompt treatment with ice packs and intravenous dantrolene and bromocriptine was instituted. Hyperpyrexia resolved completely within a few hours. Repeated blood and urine cultures were sterile. The rigidity in his lower limbs improved over a few days; he was treated with baclofen, carbidopa/levodopa, and benztropine for persistent rigidity in the upper limbs. Mr. A subsequently did well, was observed for a period of 7 days, and was discharged in stable condition.

NMS is a potentially life-threatening complication of neuroleptic therapy, with a mortality rate of $11.6 \% .^{2}$ The mecha- nism of hyperthermia in NMS is believed to be dopamine $\mathrm{D}_{2}$ receptor antagonism that results in blockage of heat-loss pathways in the anterior hypothalamus and/or increased heat production secondary to extrapyramidal rigidity. ${ }^{3}$

Risperidone, a benzisoxazole-derivative antipsychotic with high serotonin $(5-\mathrm{HT})_{2}$ receptor blockade and dose-related $\mathrm{D}_{2}$ receptor blockade, was not expected to cause NMS at low dosages. However, in recent years, numerous cases of NMS have been reported with atypical antipsychotic medications including clozapine, olanzapine, quetiapine, and risperidone. ${ }^{4}$ The results of 1 study suggested that a disturbance of serotonin metabolism may be implicated. ${ }^{5}$ Mortality has been reported to be lower in NMS associated with atypical antipsychotics. ${ }^{4}$

The patient demonstrated high fever, generalized rigidity, and altered mental state. There is considerable potential for diagnostic confusion between NMS and neurotoxicity from atypical antipsychotics because of overlap of features such as benign hyperthermia and autonomic instability. ${ }^{1}$ An infectious process should always be ruled out before a diagnosis of NMS is considered. Differentiation from serotonin syndrome is critical for successful management in cases with concomitant use of selective serotonin reuptake inhibitors (SSRIs). However, the absence of incidental use of SSRIs and the lack of myoclonus and gastrointestinal dysfunction, which are typical symptoms of serotonin syndrome, suggest that serotonin syndrome was not the diagnosis in our patient. Our patient's symptoms fully met the diagnostic criteria for NMS put forth by DSM-IV, ${ }^{6}$ Levenson, ${ }^{7}$ Pope et al., ${ }^{8}$ and Caroff and Mann. ${ }^{9}$

Most cases of risperidone-induced NMS have occurred within the first month of risperidone use, as in the case of our patient. One case series has even described the presence of a "striking, frightened facial expression" in all cases of NMS, ${ }^{10}$ as was present in our patient.

Organic brain disease and mental retardation have been identified as risk factors for NMS., ${ }^{2,10}$ The history of first-degree consanguinity and preexisting psychiatric symptoms with cognitive decline and parkinsonism exhibited by our patient may indicate the presence of a rare inherited neurodegenerative disorder such as adult neuronal ceroid lipofuscinosis (ANCL) (Kufs' disease). Studies have shown that patients with ANCL are more susceptible to NMS with the use of typical neuroleptics due to disturbances in the calcium metabolism of muscle cells. ${ }^{11}$ The loss of dopaminergic neurons in this condition may make these patients more vulnerable. Increased susceptibility to NMS has also been reported in Parkinson's disease ${ }^{12}$ and in dementia with Lewy bodies. ${ }^{13}$ Affective illness is another risk factor for NMS. ${ }^{10}$

The initial step in the treatment of NMS is the discontinuation of the causative agent. The mainstays of treatment include fluid and electrolyte replacement, reduction of temperature, and support of cardiac, respiratory, and renal functions. Pharmacotherapy with bromocriptine, dantrolene, and amantadine and electroconvulsive therapy have been described to be effective. $^{14}$ Our patient received dantrolene, bromocriptine, benztropine, carbidopa/levodopa, baclofen, and intravenous fluid replacement.

Clinicians need to be aware that NMS can occur with risperidone, despite the markedly lower incidence of extrapyramidal symptoms. In patients with neurodegenerative disorders, caution should be exercised in prescribing even atypical agents, and a high index of suspicion for NMS should be maintained. Further study is needed to reveal the mechanism of risperidone-induced NMS.

The authors report no financial affiliations or other relationships that can be considered a conflict of interest relevant to the subject of this letter. 


\section{REFERENCES}

1. Hasan S, Buckley P. Novel antipsychotics and the neuroleptic malignant syndrome: a review and critique. Am J Psychiatry 1998; 155:1113-1116

2. Shalev A, Hermesh H, Munitz H. Mortality from neuroleptic malignant syndrome. J Clin Psychiatry 1989;50:18-25

3. Mann SC, Caroff SN, Lazarus A. Pathogenesis of neuroleptic malignant syndrome. Psychiatr Ann 1991;21:175-180

4. Ananth J, Parameswaran S, Gunatilake S, et al. Neuroleptic malignant syndrome and atypical antipsychotic drugs. J Clin Psychiatry 2004;65:464-470

5. Nisijima K, Ishiguro T. Neuroleptic malignant syndrome: a study of CSF monoamine metabolism. Biol Psychiatry 1990;27:280-288

6. American Psychiatric Association. Diagnostic and Statistical Manual of Mental Disorders, Fourth Edition. Washington, DC: American Psychiatric Association; 1994

7. Levenson JL. Neuroleptic malignant syndrome. Am J Psychiatry 1985;142:1137-1145

8. Pope HG Jr, Keck PE Jr, McElroy SL. Frequency and presentation of neuroleptic malignant syndrome in a large psychiatric hospital Am J Psychiatry 1986;143:1227-1233

9. Caroff SN, Mann SC. Neuroleptic malignant syndrome. Med Clin North Am 1993;77:185-202

10. Rosebush P, Stewart T. A prospective analysis of 24 episodes of neuroleptic malignant syndrome. Am J Psychiatry 1989;146: 717-725

11. Reif A, Schneider MF, Hoyer A, et al. Neuroleptic malignant syndrome in Kufs' disease. J Neurol Neurosurg Psychiatry 2003;74: 385-387

12. Kuno S, Mizuta E, Yamasaki S. Neuroleptic malignant syndrome in parkinsonian patients: risk factors. Eur Neurol 1997;38(suppl 2): $56-59$

13. Sechi G, Agnetti V, Masuri R, et al. Risperidone, neuroleptic malignant syndrome and probable dementia with Lewy bodies. Prog Neuropsychopharmacol Biol Psychiatry 2000;24:1043-1051

14. Hall RCW, Hall RCW, Chapman M. Neuroleptic malignant syndrome in the elderly: diagnostic criteria, incidence, risk factors, pathophysiology, and treatment. Clin Geriatr 2006;14:39-46

Daniel Johnson, M.D.

Aby Z. Philip, M.D.

Dennis J. Joseph, M.D.

Roy Varghese, M.D.

Department of Internal Medicine Mary Breckinridge Hospital Hyden, Kentucky

\section{Vitamin $\mathbf{B}_{12}$ Deficiency Manifested as Mania: A Case Report}

Sir: Multiple psychiatric symptoms may precede pernicious anemia. Only 1 case of full manic syndrome secondary to $B_{12}$ deficiency has been reported. ${ }^{1}$

Case report. Ms. A was a 35-year-old woman with no personal or family history of psychiatric disorders, who was admitted to the hospital in February of 2005 with a 3-week history of episodes of manic symptoms of several hours' duration, including grandiosity, hyperactivity, sexual indiscretion, hyperphagia, irritable mood, reckless behavior, flight of ideas, and overbearing manner. These episodes had a sudden onset and conclusion, and the patient was able to remember them. No hallucinations were reported at any time.

At admission, she was oriented in all spheres, but was easily distractible. Full manic syndrome (DSM-IV criteria) was present and there was no coexisting delirium. All these psychiatric symptoms disappeared after 1 hour, without any medical intervention.

An examination revealed the presence of a left Babinski's reflex and ataxia, as well as a bilateral loss of proprioception, vibration, and sensation of light touch and pain in the lower extremities. Strength and reflex in the lower extremities were diminished

The results of general blood chemistries, a thyroid function test, and a comprehensive drug screen were within normal limits. Although the results of electroencephalogram (EEG) and brain magnetic resonance imaging were normal, the duration, as well as the sudden onset and conclusion of episodes, suggested an epileptic cause for this patient's mania. ${ }^{2}$ Ms. A was treated with valproic acid for 3 weeks with no improvement.

Serum vitamin $\mathrm{B}_{12}$ level was measured and was found to be markedly diminished-less than $60 \mathrm{pg} / \mathrm{mL}$. Vitamin $B_{12}(1000$ $\mu \mathrm{g} /$ day) was administered intravenously for 1 week. After the third day of $\mathrm{B}_{12}$ replacement therapy, our patient did not experience new manic episodes. Therapy was continued with 1000 $\mu \mathrm{g}$ intramuscular injections weekly for 1 month and then with monthly intramuscular injections as maintenance treatment. Within 1 month, neurologic symptoms had improved. One year after $\mathrm{B}_{12}$ vitamin replacement started, her mental status was still normal.

Although many psychiatric symptoms have been considered to be related to $\mathrm{B}_{12}$ deficiency, ${ }^{3,4}$ this is the first report of short duration episodes of manic symptoms secondary to $\mathrm{B}_{12}$ deficiency. The absence of previous psychiatric or medical disorders, coupled with her continued clinical remission while she was receiving $\mathrm{B}_{12}$ alone, argues that $\mathrm{B}_{12}$ deficiency was the most likely cause of her psychiatric disorder.

Our case could also raise the hypothesis of different neurobiological mechanisms underlying mood disorders and the importance of $\mathrm{B}_{12}$ and folate in their genesis. ${ }^{5}$

The authors report no financial affiliations or other relationships relevant to the subject of this letter.

\section{REFERENCES}

1. Goggans FC. A case of mania secondary to vitamin B12 deficiency. Am J Psychiatry 1984;141:300-301

2. Duane DD, Ettinger AB, Reed ML, et al. Prevalence of bipolar symptoms in epilepsy vs other chronic health disorders [letter]. Neurology 2006;66:954

3. Zucker DK, Livingston RL, Nakra R, et al. B12 deficiency and psychiatric disorders: case report and literature review. Biol Psychiatry 1981;16:197-205

4. Verbanck PMP, Le Bon O. Changing psychiatric symptoms in a patient with vitamin B12 deficiency [letter]. J Clin Psychiatry 1991; $52: 182-183$

5. Tiemeier H, van Tuijl HR, Hofman A, et al. Vitamin B12, folate, and homocysteine in depression: the Rotterdam Study. Am J Psychiatry 2002;159:2099-2101

German Jorge Gomez-Bernal, M.D. Rehabilitation Unit Psychiatric Hospital of Teruel Teruel, Spain Milagros Bernal-Perez, Ph.D. University of Zaragoza Zaragoza, Spain 


\section{Case Report: Bupropion-Induced Claustrophobia}

Sir: Although emergent anxiety can occur with bupropion therapy $\left(5 \%-6 \%\right.$ per the package insert $\left.{ }^{1}\right)$, to date there are no reports of claustrophobia associated with the drug. We present a case of new-onset claustrophobia related to bupropion therapy.

Case report. Mr. A, a 59-year-old man with no prior psychiatric history, was prescribed bupropion in May of 2005 for a major depressive episode (DSM-IV criteria). Target symptoms included decreased energy, feelings of worthlessness, decreased appetite, and restlessness. Past treatment consisted of sertraline (taken from December 2004 to January 2005), discontinued due to sexual side effects, and citalopram (prescribed once in March 2005), discontinued for lack of efficacy.

The patient was administered bupropion SR, titrated to 150 $\mathrm{mg}$ twice daily. Soon after, he self-decreased his dose to $150 \mathrm{mg}$ daily because of irritability and "claustrophobia," described as an intense feeling that the walls were closing in. He also reported increased heart rate, diaphoresis, and difficulty breathing while in rooms with closed doors, in elevators, in the shower, and in the back seat of compact cars. These symptoms were not present in situations that did not involve enclosed spaces. In clinic, he insisted the office door be left open. Bupropion was subsequently discontinued (June 29, 2005) and venlafaxine was initiated on the same day. After 1 month, his claustrophobic symptoms resolved. Nine months after bupropion therapy was terminated, anxiety symptoms have not recurred.

Claustrophobia is an anxiety disorder that involves the fear of confined or enclosed spaces. ${ }^{2}$ Although one of the most prevalent specific phobias in the general population, only $20 \%$ of those affected will seek medical attention. ${ }^{3}$ Thus, the disorder often goes undiagnosed.

Although the exact pathophysiology of claustrophobia is unknown, there is compelling evidence that supports the role of the noradrenergic system in anxiety disorders. The activation of brain noradrenergic neurotransmission in the presence of acute stress results in neuroendocrine, autonomic, behavioral, and cognitive responses. ${ }^{4,5}$ Unlike other agents, bupropion exerts its therapeutic effects by inhibiting norepinephrine and dopamine reuptake.

Because of its actions on the noradrenergic system, it is reasonable that bupropion may worsen anxiety. In a study by Ashton and Rosen, ${ }^{6} 15 \%$ of patients treated with bupropion for sexual side effects associated with selective serotonin reuptake inhibitors discontinued therapy due to anxiety or tremor. Additionally, Young ${ }^{7}$ reported a case of panic occurring following the addition of bupropion to fluoxetine.

To our knowledge this is the first report of bupropioninduced claustrophobia. We recommend caution with the use of bupropion in patients with primary anxiety disorders.

Dr. Mascarenas has received grant/research support from Forest Laboratories. Dr. Malhotra reports no financial affiliations or other relationships relevant to the subject of this letter.

The opinions contained in this letter are those of the authors and are not to be construed as official or reflecting the views of the South Texas Veterans Health Care System.

\section{REFERENCES}

1. Wellbutrin SR [package insert]. Research Triangle Park, NC: GlaxoSmithKline; 2006
2. American Psychiatric Association. Diagnostic and Statistical Manual of Mental Disorders, Fourth Edition, Text Revision. Washington, DC: American Psychiatric Association; 2000

3. Fyer AJ. Current approaches to etiology and pathophysiology of specific phobia. Biol Psychiatry 1998;44:1295-1304

4. Brunello N, Blier P, Judd LL, et al. Noradrenaline in mood and anxiety disorders: basic and clinical studies. Int Clin Psychopharmacol 2003;18:191-202

5. Sullivan GM, Coplan JD, Kent JM, et al. The noradrenergic system in pathological anxiety: a focus on panic with relevance to generalized anxiety and phobias. Biol Psychiatry 1999;46:1205-1218

6. Ashton AK, Rosen RC. Bupropion as an antidote for serotonin reuptake inhibitor-induced sexual dysfunction. J Clin Psychiatry 1998;59: $112-115$

7. Young SJ. Panic associated with combining fluoxetine and bupropion [letter]. J Clin Psychiatry 1996;57:177-178

Jodie V. Malhotra, Pharm.D. University of the Incarnate Word South Texas Veterans Health Care System San Antonio, Texas Cynthia A. Mascarenas, Pharm.D., M.S., B.C.P.P. South Texas Veterans Health Care System University of Texas Health Science Center San Antonio, Texas

\section{Is There an Alternative to Seclusion or Mechanical Restraint?}

Sir: The treatment of agitated patients has been reviewed in the literature. ${ }^{1}$ We would like to comment on the use of restraints and seclusion for such patients. We agree that mechanical restraints or seclusion should be the last measure used in the majority of cases, but think that application of such methods is sometimes necessary. A decline in the use of seclusion or mechanical restraint due to administrative regulation might be dangerous and the reason for increased violence on psychiatric wards. ${ }^{2}$ Furthermore, some patients have been known to request placement in seclusion ${ }^{3}$ or to be mechanically restrained. Although significant literature exists, there is a lack of knowledge about patients' attitudes toward these methods independent of personal experiences.

Method. Attitudes of currently hospitalized patients with schizophrenia toward use of mechanical restraints (seclusion is a rare practice in Croatia) were examined. We constructed a questionnaire consisting of questions mostly related to patients' emotional reactions toward use of mechanical restraints and to the phenomenon of aggression on psychiatric wards. The participants gave consent to participate in the study after the purpose was explained. Data were collected in August 2006. Although 120 patients agreed to participate in the assessment, 13 patients $(11 \%)$ refused further participation during assessment and $36(30 \%)$ failed to provide analyzable data because of poor psychiatric states. Analyzable data were collected from 71 patients $(59 \%)($ male $=54$, female $=17 ;$ mean $[S D]$ age $=41.47$ [12.62] years, range, 19-70 years; mean [SD] duration of disorder $=12.70$ [9.03] years; 11 patients had been treated on "acute wards," 23 on "chronic wards," and 37 at the Department of Forensic Psychiatry).

Results. Of the final sample, 61 patients $(86 \%)$ had the opinion that mechanical restraint was sometimes necessary, and 42 $(59 \%)$ had the attitude that mechanical restraint of another aggressive patient reduced their feeling of insecurity. Thirty-two 
patients $(45 \%)$ had been mechanically restrained 1 or more times. Of those who had been mechanically restrained, 17 $(53 \%)$ had the attitude that, in their case, restraint was, at least once, necessary; 11 (34\%) requested restraints 1 or more times; and $12(38 \%)$ had experienced feelings of being safer during restraint.

We agree that, in terms of clinical guidelines, mechanical restraints or seclusion should be used for the least amount of time possible. Yet, use of restraints influences the "atmosphere on a ward." In accordance with our results, decreasing feelings of insecurity or danger, which are present in a significant number of patients on the psychiatric ward, is part of that "atmosphere."

Furthermore, we think that, in some cases, fortunately rare, feelings of insecurity among patients in the ward might become the reason for prolonged use of seclusion or mechanical restraint. For example, hostile attack of a patient by another patient might lead to increased feelings of insecurity among other patients on the ward. Feelings of insecurity among the other patients might be so intense that, independent of the psychiatric state of the patient who made the attack, seclusion or restraints should be used for longer periods of time. In similar cases, these methods might paradoxically protect the restrained patient from his or her environment. We do believe that such situations are more frequent on forensic wards, but are possible on all kinds of wards.

The authors report no financial or other relationships relevant to the subject of this letter.

\section{REFERENCES}

1. Marder SR. A review of agitation in mental illness: treatment guidelines and current therapies. J Clin Psychiatry 2006;67(suppl 10): $13-21$

2. Khadivi AN, Patel RC, Atkinson AR, et al. Association between seclusion and restraint and patient-related violence. Psychiatr Serv 2004;55:1311-1312

3. Meehan T, Vermeer C, Windsor C. Patients' perceptions of seclusion: a qualitative investigation. J Adv Nurs 2000;31:370-377

Branimir Margetic, M.D. Branka Aukst-Margetic, M.D., M.S. Adela Matušin, R.N. Neuropsychiatric Hospital "Dr. Ivan Barbot" Popovača, Croatia

\section{Massive Epistaxis and Subconjunctival Hemorrhage Due to Combination of Paroxetine and Limaprost Alfadex: A Case Report}

Sir: A patient presented to us having experienced a few days of massive epistaxis and hyposphagma (severe hemorrhage into the conjunctiva) while taking paroxetine. Pharmaceutical use was also notable for the peripheral vasodilator limaprost alfadex. To our knowledge, no such case has been reported in the literature. We believe the combination of paroxetine and limaprost alfadex led to the bleed.

Case report. Ms. A, a 66-year-old woman with blood counts and blood coagulation tests within normal limits, presented in June 2005 to the orthopedic surgery department with com- plaints of coldness of limbs, continuing lower-extremity pain, insomnia, and loss of appetite. She underwent a nerve-root block for lumbar spinal canal stenosis and started limaprost alfadex $15 \mu \mathrm{g} /$ day. Limaprost alfadex, an oral prostaglandin $E_{1}$ derivative, is frequently used in Japan for the treatment of peripheral circulatory disturbances. Because the symptoms overall did not improve, she was referred to the psychiatry service (July 2005). She had lost $10 \mathrm{~kg}$ in 2 months and complained strongly of anxiety and frustration due to inability to do housework and to memory loss. She was admitted in September 2005.

The patient was diagnosed with DSM-IV major depressive disorder with psychotic features based on hypochondriacal and persecutory delusions and aggression. When she refused food and medication due to delusions of being poisoned, electroconvulsive therapy (ECT) was introduced (October-November 2005 , total of 11 treatments). ECT was effective, and she began to take medication again after the sixth ECT treatment. She was restarted on limaprost alfadex treatment $(15 \mu \mathrm{g} / \mathrm{day})$. When ECT was completed in early November, paroxetine $20 \mathrm{mg}$ /day was started, increasing to $40 \mathrm{mg} /$ day after 2 days and to 50 $\mathrm{mg} /$ day after 4 days. The patient's mood remained normal.

On the 29th and 30th days after starting paroxetine, she experienced massive epistaxis (more than $500 \mathrm{~mL}$ per episode). On the 30th day of treatment, paroxetine was decreased to $40 \mathrm{mg} /$ day, but similar epistaxis recurred the next day. An otolaryngologist determined that hemorrhage was from the right Kiesselbach plexus; however, no other abnormality was observed. Complete blood count and blood coagulation findings were within normal limits. On the 36th day of paroxetine treatment, limaprost alfadex was reduced from $15 \mu \mathrm{g} /$ day to $10 \mu \mathrm{g} /$ day. No further epistaxis episodes occurred. Upper (day 45 of paroxetine treatment) and lower (day 50 of paroxetine treatment) gastrointestinal endoscopy were performed, and no hemorrhage was observed. Because of intermittent complaints of lower extremity coldness and pain, the dosage of limaprost alfadex was returned to $15 \mu \mathrm{g} /$ day. Since January 2006, prescriptions for paroxetine $40 \mathrm{mg} /$ day and limaprost alfadex 15 $\mu \mathrm{g} /$ day have continued, and the patient has been followed at the outpatient clinic. There has been no recurrent epistaxis as of September 2006. However, right eye hyposphagma was recently observed at a psychiatry clinic appointment. If this continues, we will consider decreasing paroxetine to $30 \mathrm{mg} /$ day.

Mild epistaxis during selective serotonin reuptake inhibitor (SSRI) treatment is a well-known rare adverse effect, but our patient's epistaxis was severe. Hyposphagma with paroxetine might be very rare. ${ }^{1}$ Since only a few case reports ${ }^{2}$ have suggested SSRI use alone can trigger even mild bleeding, including ecchymoses, purpura, and epistaxis, we cannot make a strong conclusion. After carefully examining the drugs in concurrent use for our patient, it is quite possible that limaprost alfadex, in combination with paroxetine, contributed to severe epistaxis and hyposphagma.

When patients present with severe symptoms, including psychotic features, many clinicians use relatively high doses of antidepressants for continuation and maintenance treatment. ${ }^{3}$ Because vasodilating prostaglandins such as limaprost alfadex are used frequently in fields such as orthopedic surgery, it is important that clinicians know there may be a tendency for hemorrhage when an SSRI is also used; physicians should consider decreasing the SSRI maintenance dose in such instances.

The authors report no financial affiliation or other relationship relevant to the subject of this letter. 


\section{REFERENCES}

1. Wilmshurst PT, Kumar AV. Subhyaloid haemorrhage with fluoxetine [letter]. Eye 1996;10:141

2. Ottervanger JP, Stricker BH, Huls J, et al. Bleeding attributed to the intake of paroxetine. Am J Psychiatry 1994;151:781-782

3. Franchini L, Gasperini M, Perez J, et al. Dose-response efficacy of paroxetine in preventing depressive recurrences: a randomized, double-blind study. J Clin Psychiatry 1998;59:229-232

Nobuhiro Sugiyama, M.D., Ph.D. Daimei Sasayama, M.D. Naoji Amano, M.D., Ph.D. Department of Neuropsychiatry Shinshu University School of Medicine Matsumoto, Nagano, Japan

\section{The Tolerability of Oral-Loaded Valproate After Remission of Acute Mania in Japanese Patients With Bipolar Disorder}

Sir: It has been reported by McElroy et al. ${ }^{1}$ that the onset of the antimanic effects of valproate can be accelerated by administering valproate at a loading dose of $20 \mathrm{mg} / \mathrm{kg} / \mathrm{day}$ and that doing so produces a rapid response with minimal side effects. Since then, several studies ${ }^{2,3}$ have shown that oral-loaded divalproex is an effective and well-tolerated treatment in patients with acute mania. However, these previous studies were limited to analysis of acute treatment effects. The tolerability of continuing the initial dose of valproate after the remission of mania has yet to be established. We conducted a naturalistic observational study to assess the safety of continuous treatment with valproate at high doses in bipolar patients.

Method. The study included 9 patients, aged 24 to 79 years (7 female, 2 male), who presented for the treatment of acute mania to the Hiroshima University hospital from July 2002 through December 2004. All patients were diagnosed with acute mania associated with bipolar I disorder according to DSM-IV criteria. Two were diagnosed with rapid-cycling bipolar disorder. The nature and purpose of the study were explained, and informed consent was obtained from all patients. The study was conducted according to a protocol approved by the Ethics Committee of the Hiroshima University School of Medicine. Patients were required to have manic symptoms of sufficient severity to have Young Mania Rating Scale (YMRS) ${ }^{4}$ scores of 23 or more. Remission was defined as achieving YMRS scores under 8 . The mean \pm SD YMRS score at baseline was $34.6 \pm$ 5.1. Other psychotropics (antipsychotics and benzodiazepines) were allowed for the treatment of agitation or insomnia if necessary. Valproate was administered at $20 \mathrm{mg} / \mathrm{kg} /$ day or 30 $\mathrm{mg} / \mathrm{kg} /$ day for at least 4 weeks. The mean YMRS scores were analyzed by Scheffe's multiple comparison test following repeated-measures analysis of variance.

Results. All 9 patients received valproate treatment at the initial dose for at least 4 weeks. The mean valproate loading dose was $1360 \mathrm{mg} /$ day. Serum levels of valproate were greater than $50 \mu \mathrm{g} / \mathrm{mL}$ by day 4 in all patients. All patients showed significant improvement, and the mean \pm SD YMRS score was $21.4 \pm 4.5$ at the end of week $1(p<.0001)$. At the fourth week, all achieved remission, and their mean \pm SD YMRS score was $3.0 \pm 2.4$. The mean serum level of valproate after the patients' mania had stabilized was about $95 \mu \mathrm{g} / \mathrm{mL}$. No adverse events were reported during the first 4 weeks of treatment. The results confirmed that oral loading of valproate for up to 4 weeks is a safe and effective treatment for Japanese patients with acute mania.

However, by week 12,8 of 9 patients had reported adverse effects, including sedation ( 8 patients), nausea ( 1 patient), and weight gain (1 patient), and 8 patients had their dose reduced as a consequence. Three patients who were followed beyond the 12 -week follow-up period discontinued valproate because of adverse effects.

Previous studies ${ }^{1-3}$ showed that oral loading with valproate for 10 days or less is well tolerated in the majority of patients with acute mania. Our study demonstrates that continuing highdose valproate may cause adverse effects in Japanese patients with bipolar disorder. Thus, the doses of valproate may need to be decreased after remission. Further controlled examinations are needed to ascertain whether it is necessary to decrease the dose after remission and whether a lower dose of valproate is effective for maintenance treatment.

This study was supported by the Research Grants for Nervous and Mental Disorders from the Ministry of Health, Labor and Welfare, Japan, in 2005 .

The authors report no additional financial relationship or affiliation relevant to the subject of this letter.

\section{REFERENCES}

1. McElroy SL, Keck PL, Tugrul KC, et al. Valproate as a loading treatment in acute mania. Neuropsychobiology 1993;27:146-149

2. Hirschfeld RMA, Baker JD, Wozniak P, et al. The safety and early efficacy of oral-loaded divalproex versus standard-titration divalproex, lithium, olanzapine, and placebo in the treatment of acute mania associated with bipolar disorder. J Clin Psychiatry 2003;64: 841-846

3. Oluboka OJ, Bird DC, Kutcher S, et al. Pilot study of loading versus titration of valproate in the treatment of acute mania. Bipolar Disord 2002;4:341-345

4. Young RC, Biggs JT, Ziegler VE, et al. A rating scale for mania: reliability, validity, and sensitivity. Br J Psychiatry 1978;133:429-435

Kiyomi Shinohara, M.D. Osaka Institute of Psychiatry Shin-Abuyama Hospital Osaka, Japan

Yasumasa Okamoto, M.D., Ph.D. Hiroaki Jitsuiki, M.D., Ph.D.

Hidehisa Yamashita, M.D., Ph.D. Shigeru Morinobu, M.D., Ph.D. Shigeto Yamayaki, M.D., Ph.D. Department of Psychiatry and Neurosciences Hiroshima University Hiroshima, Japan 